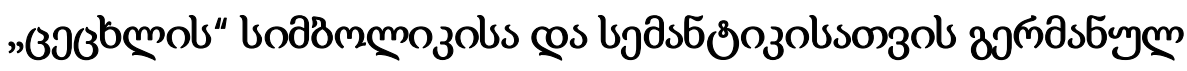

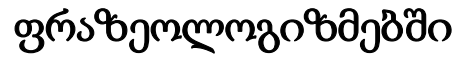

\author{
аmegäsdj colsbs

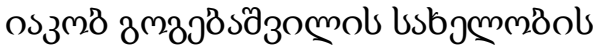

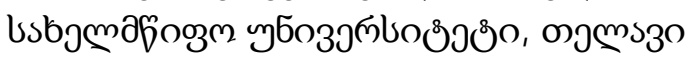 \\ https://doi.org/10.52340/idw.2021.510
}

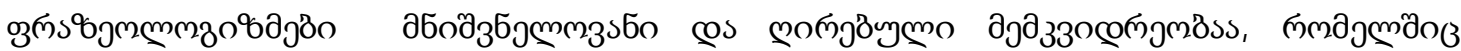

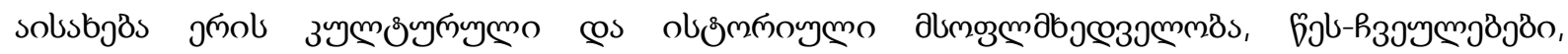

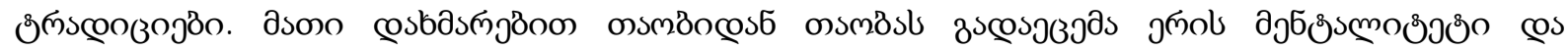

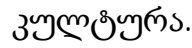

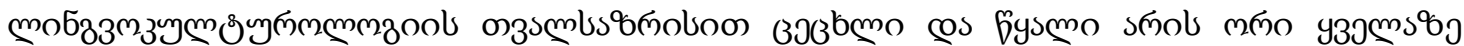

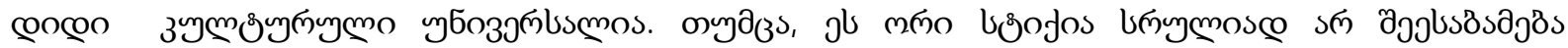

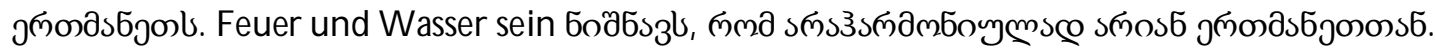

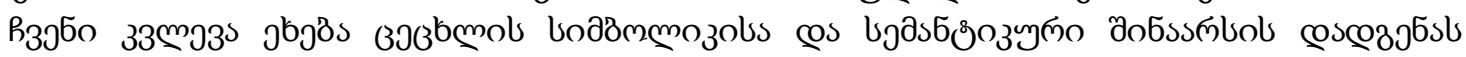

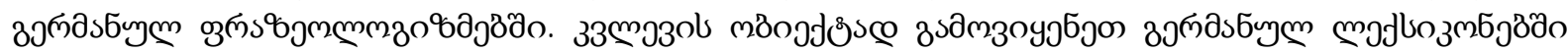

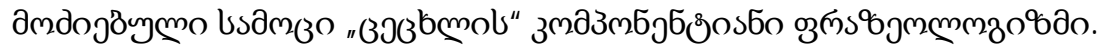

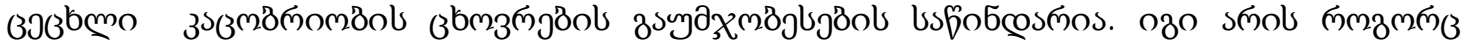

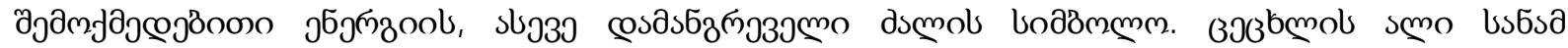

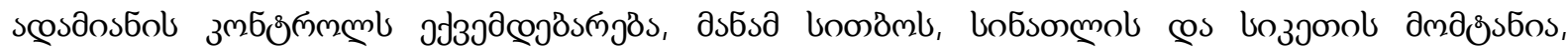

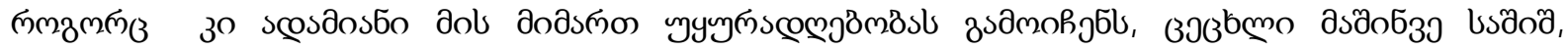

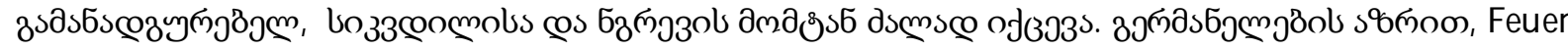

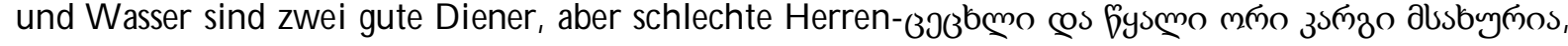

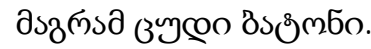

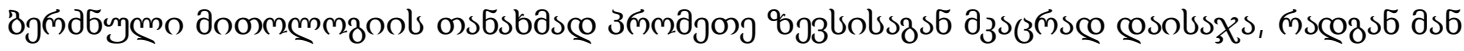

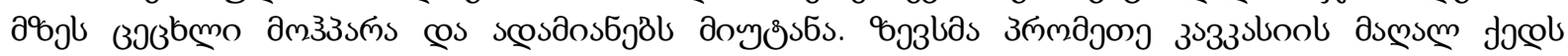

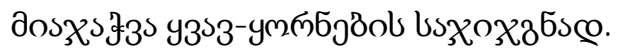

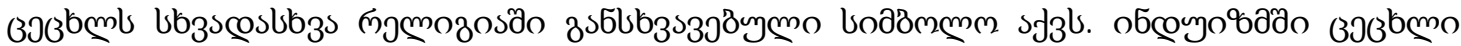

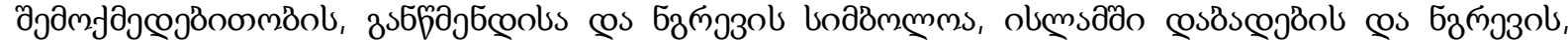

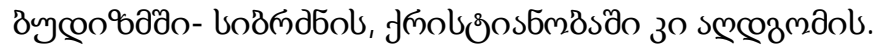

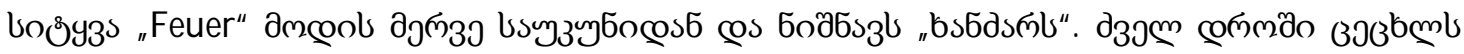

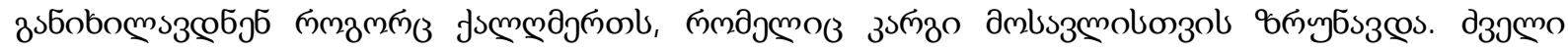

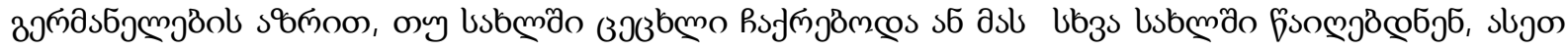

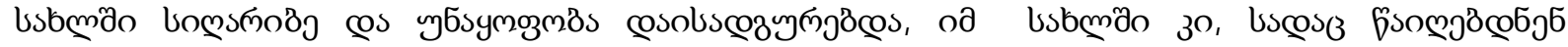

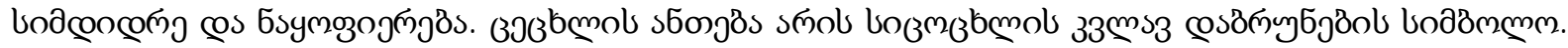

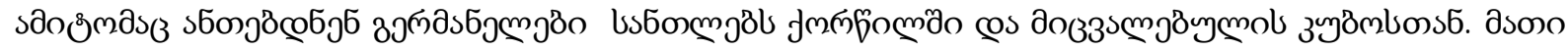

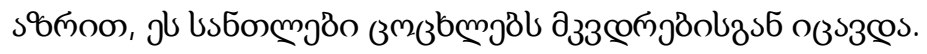

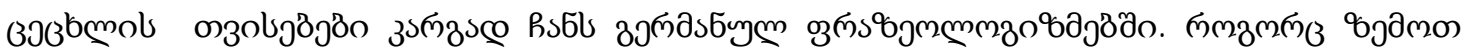

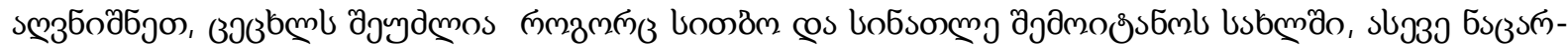

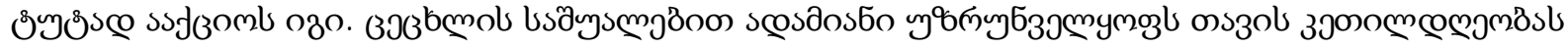

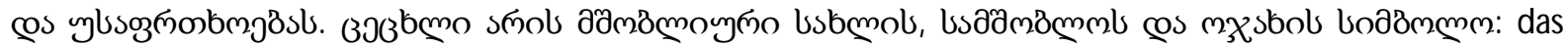

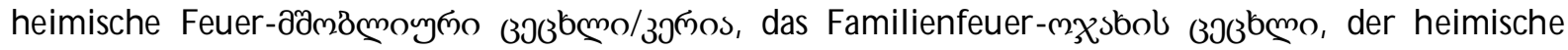




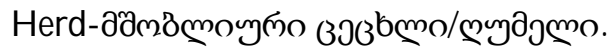

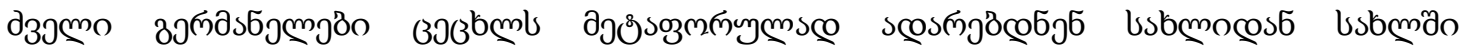

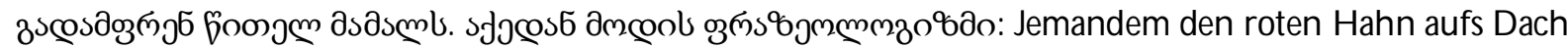

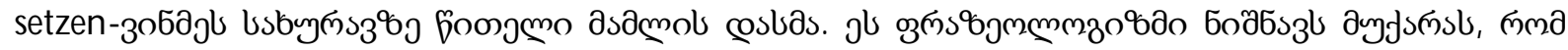

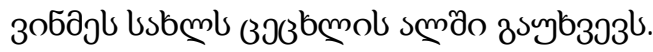

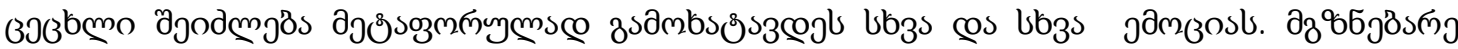

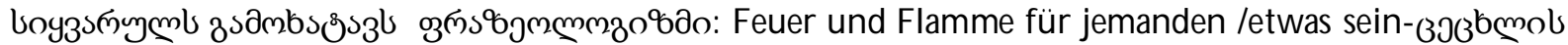

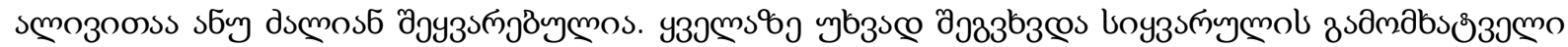

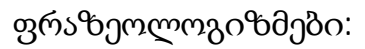

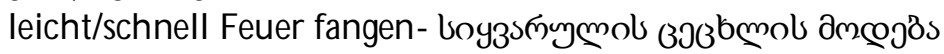

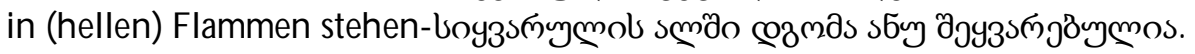

Feuer der Liebe-boyzsmingob $393^{6} \mathrm{mo}$

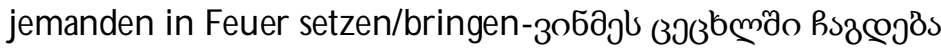

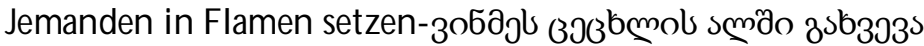

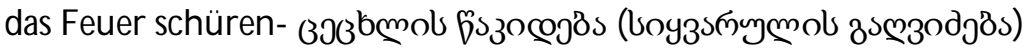

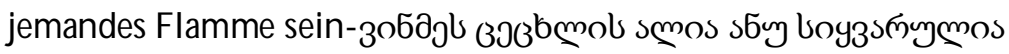

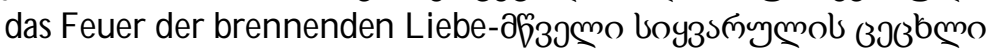

Die Liebe brennt wie das Feuer im Herzen-boyzsnиm з з

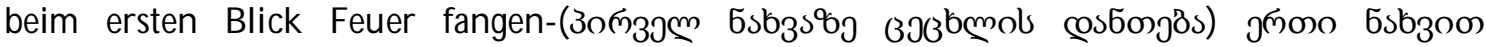

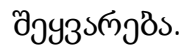

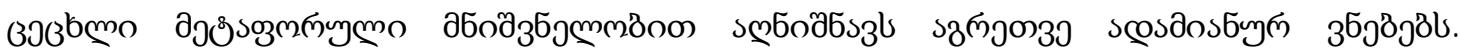

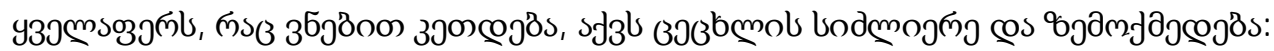

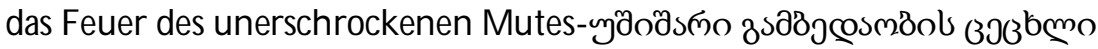

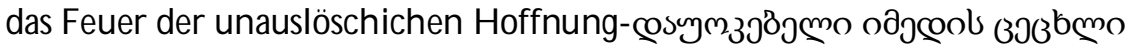

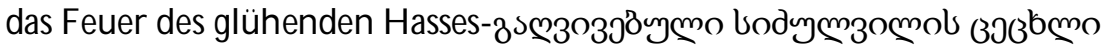

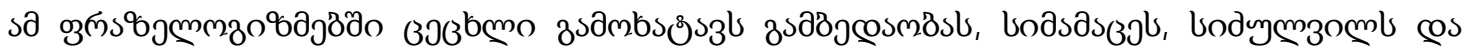

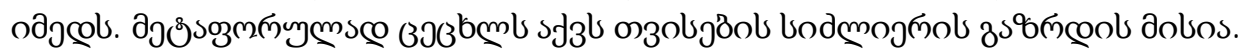

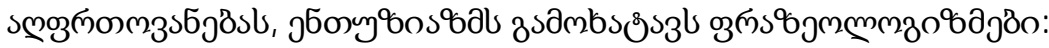

in Feuer kommen/geraten-

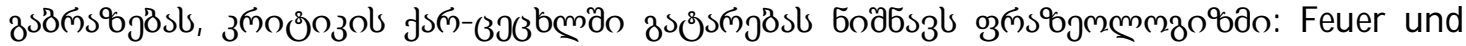
Flamme speien/spucken- 3936 mol cos smol ozynombjös.

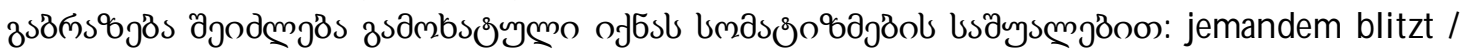

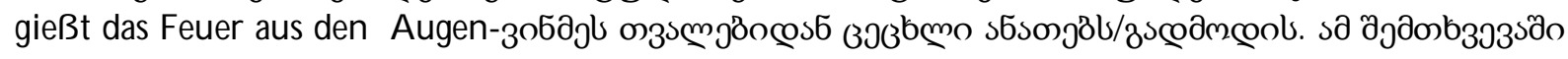

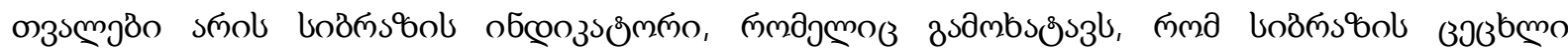

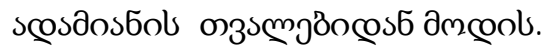

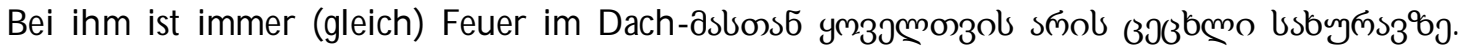

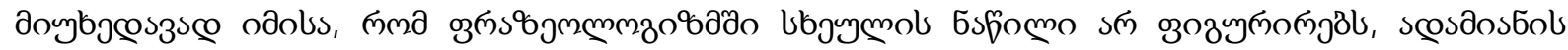

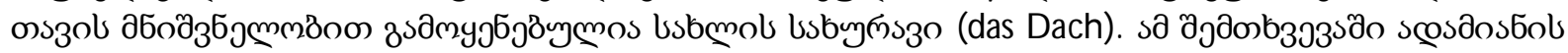

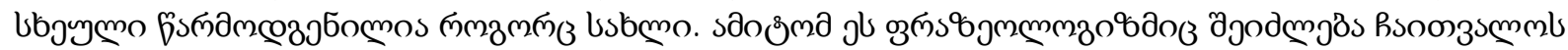

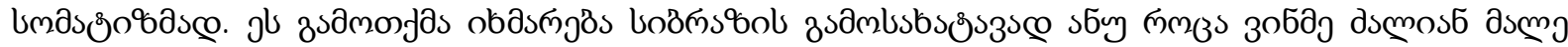

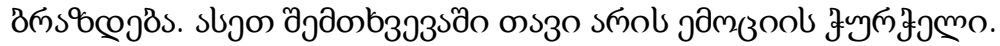

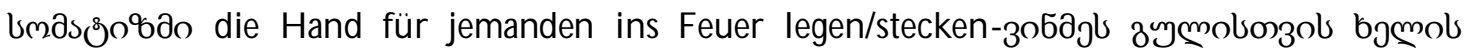

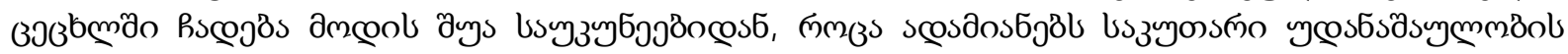

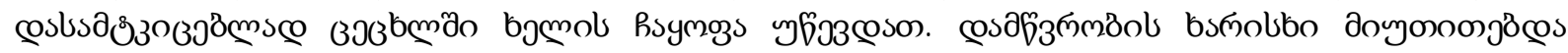

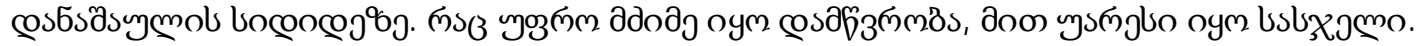

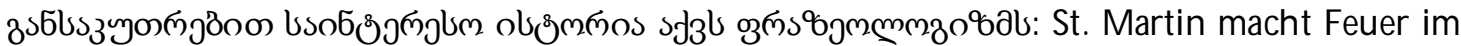

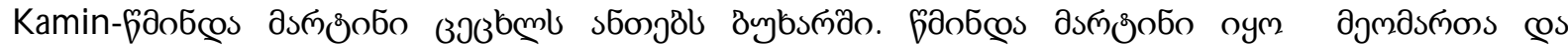

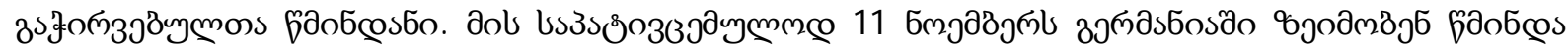

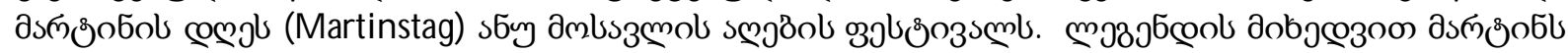

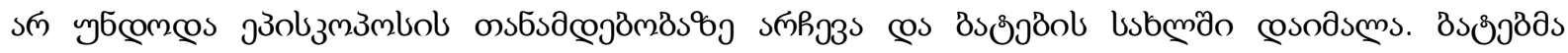




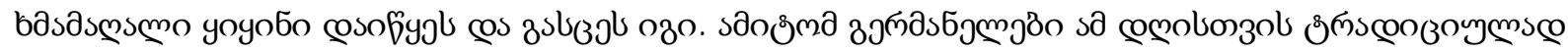

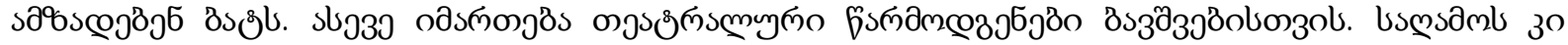

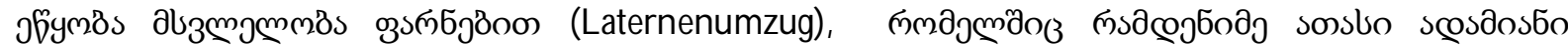
дмбsfoмm

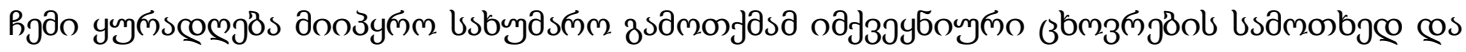

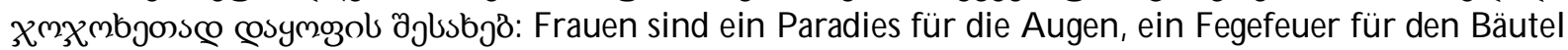

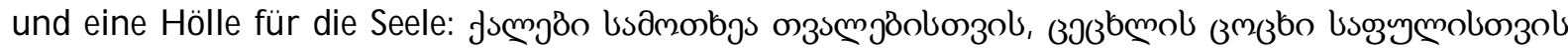
cos xmxabjon bymolsongol.

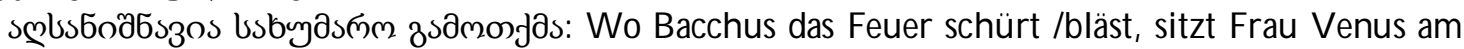

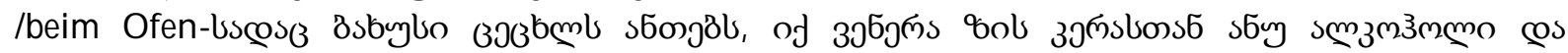

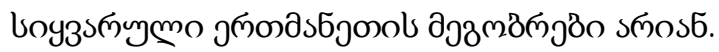

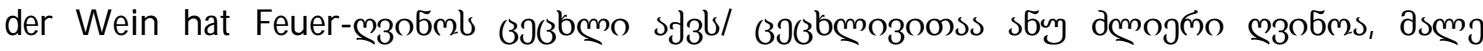
sonмmठl scosanosbl.

Gebranntes Kind scheut das Feuer- cos

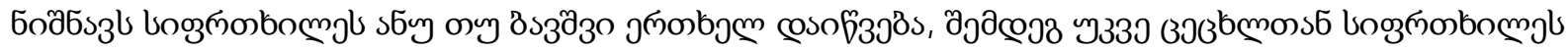

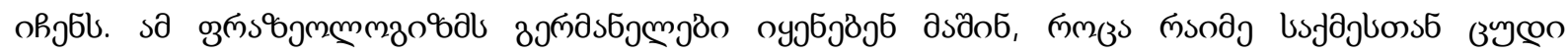

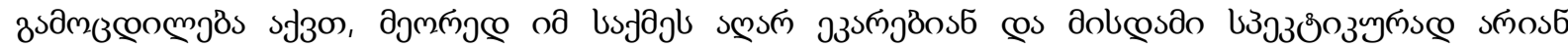
зsб6умдом⿻о.

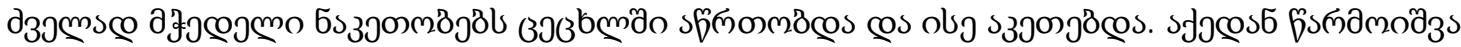

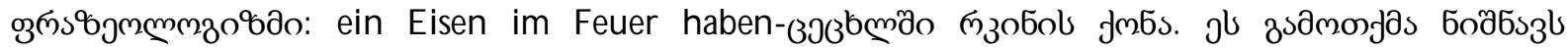

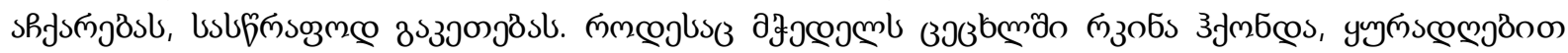

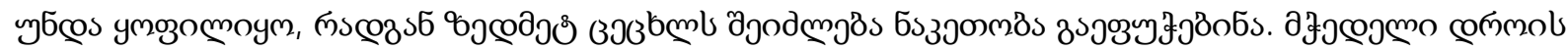

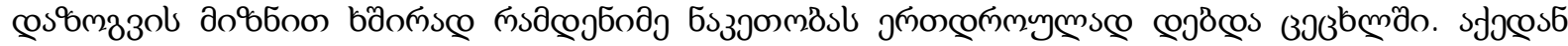

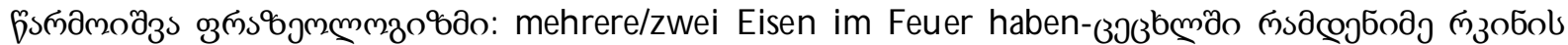

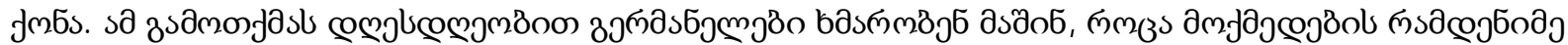

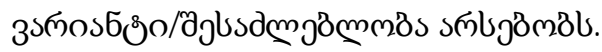

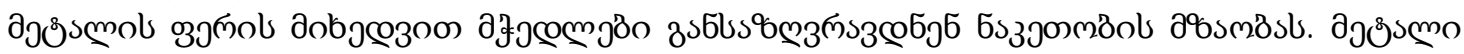

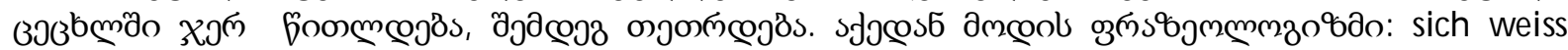

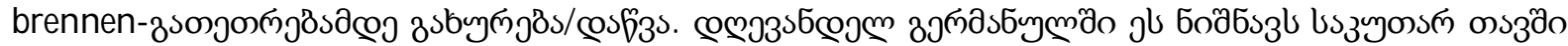

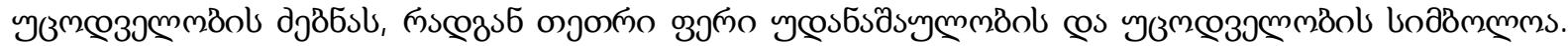

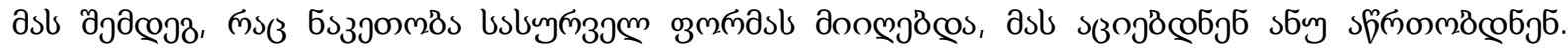

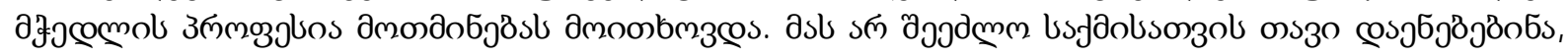

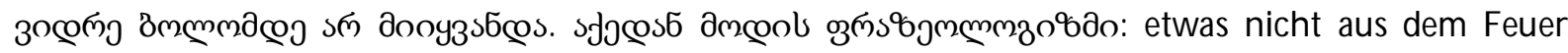

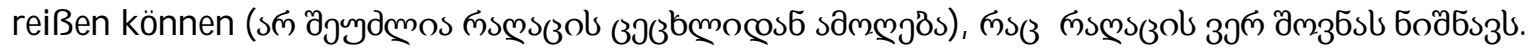

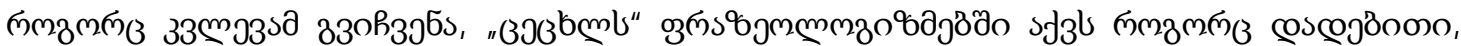

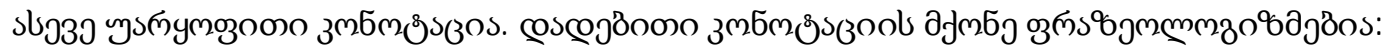

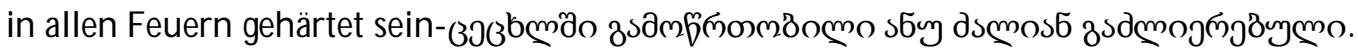

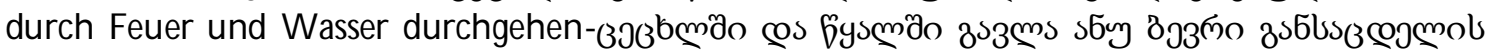
zscososts cos zudmojmjos.

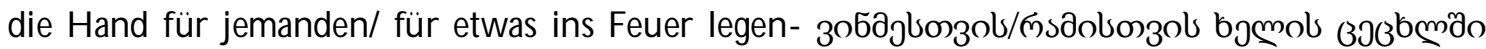

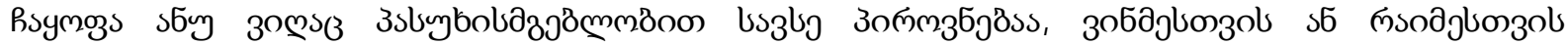

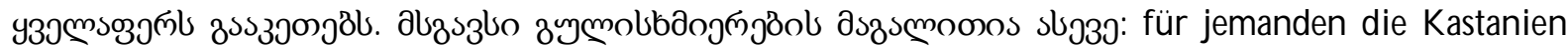

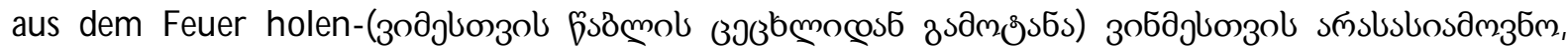

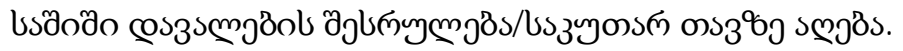

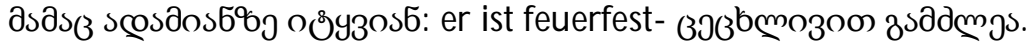

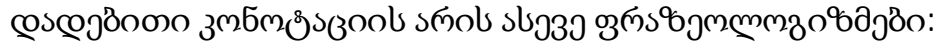

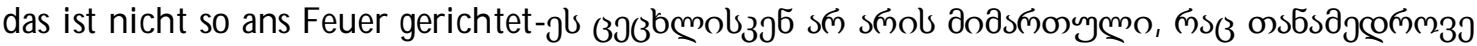

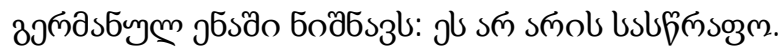

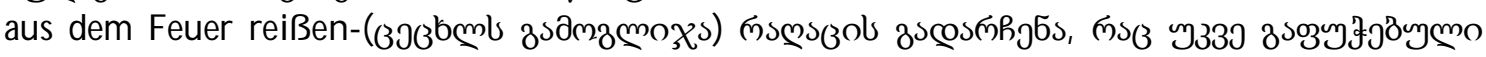
oym. 


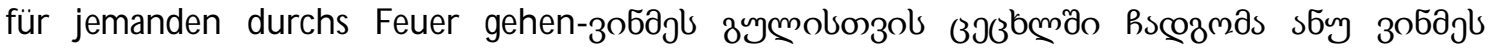

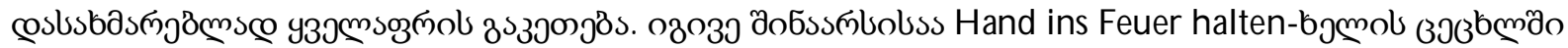
‡ons.

Er ist Feuer und Flamme (

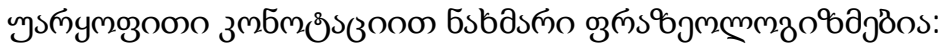

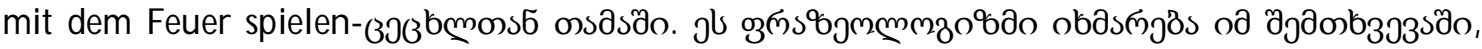

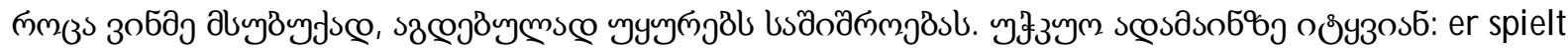

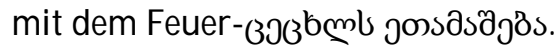

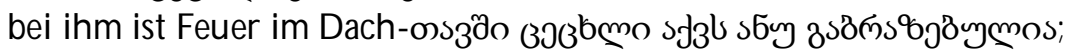

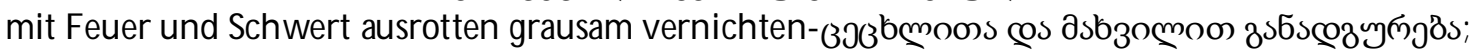

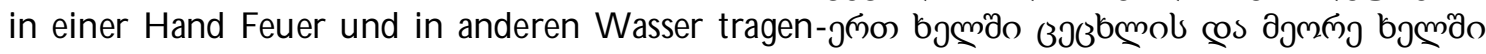

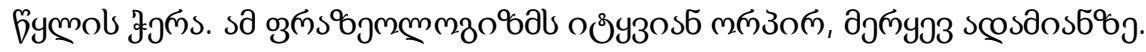

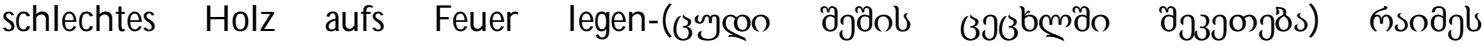

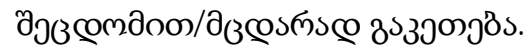

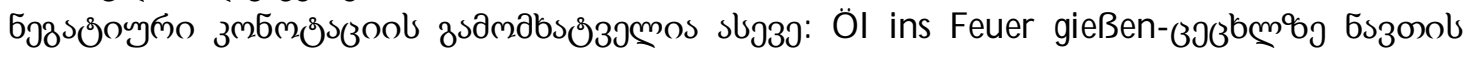

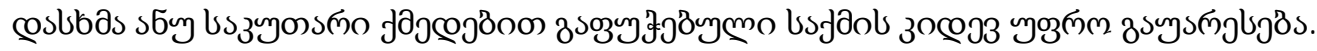

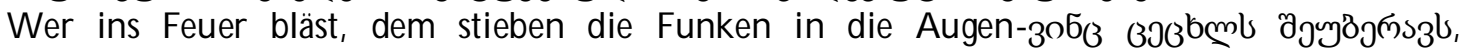

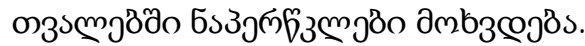

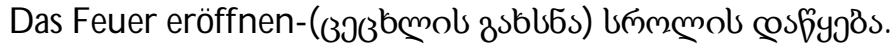

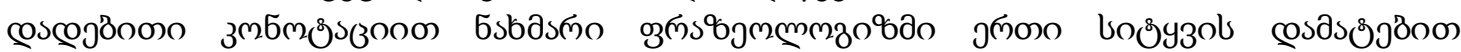

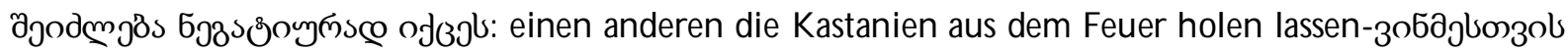

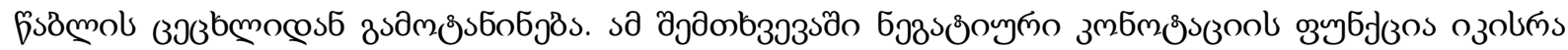

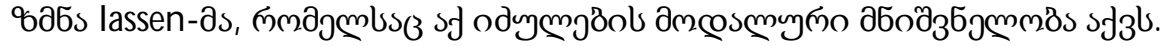

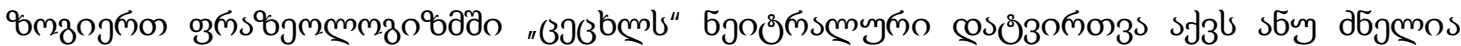

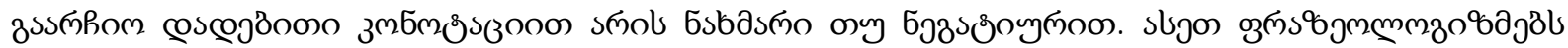

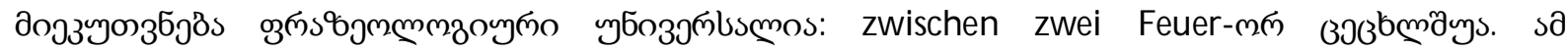

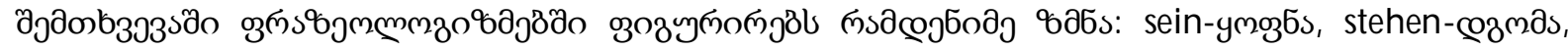

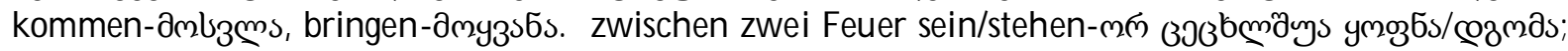

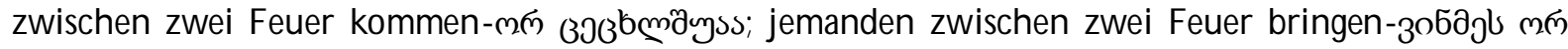

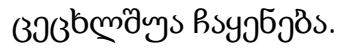

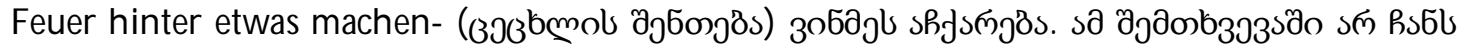

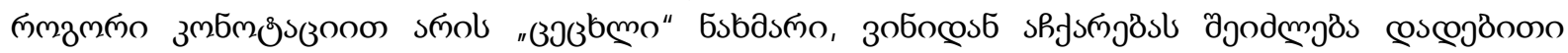

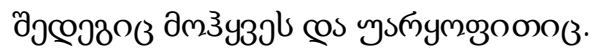

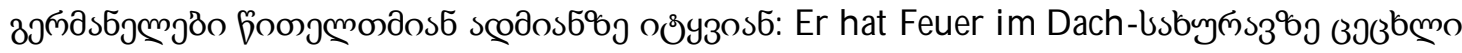

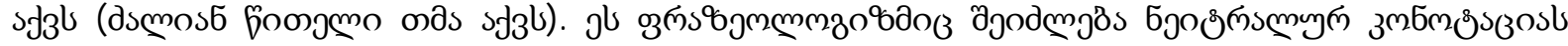

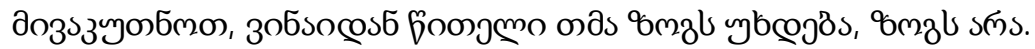

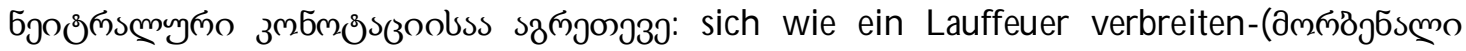

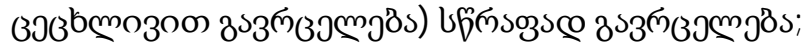

Das Feuer, das mich nicht brennt, lösch ich nicht- $3 \sigma^{6}$ उऽनऊмठे.

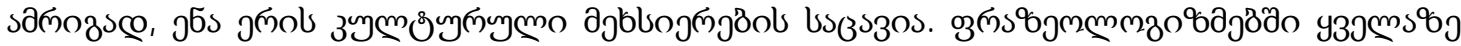

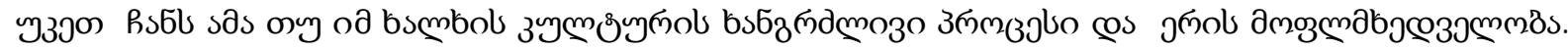

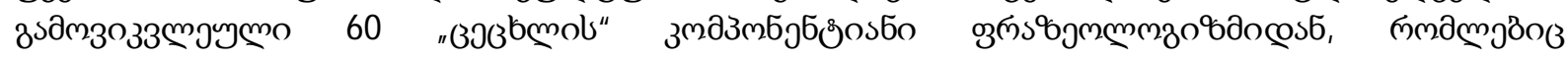

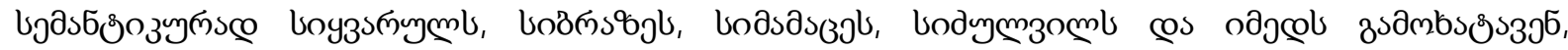

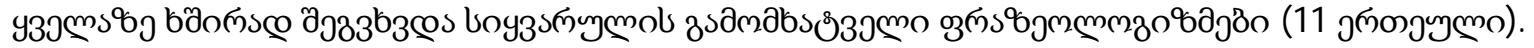

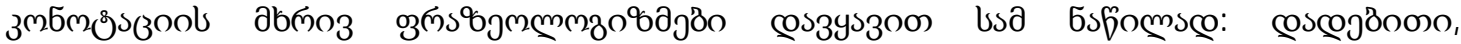

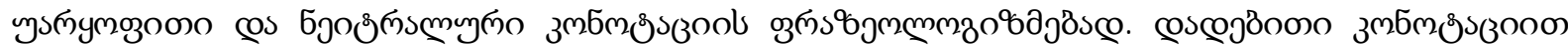

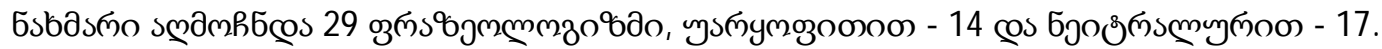

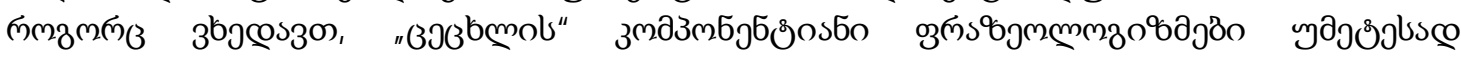

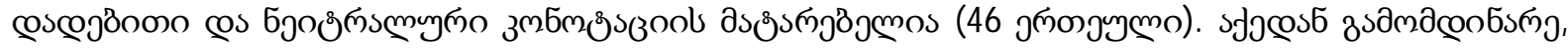




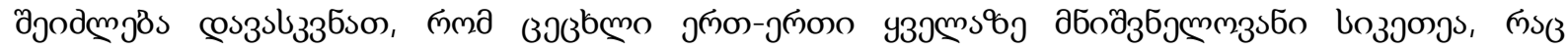

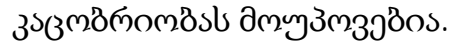

\title{
zozmomżnsogos:
}

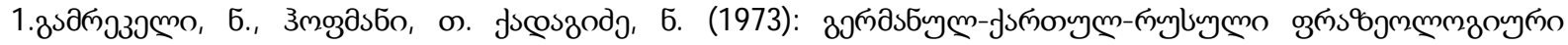

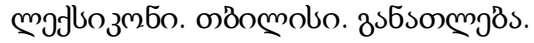

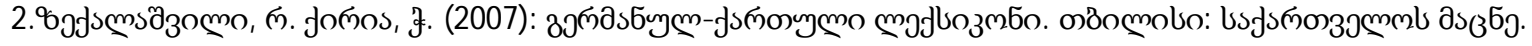

3.Götz, D. u. a.:(2008) Droßwörterbuch Deutsch als Fremdsprache; Langenscheidt-Berlin- München -Wien -Zürich New York

4.Duden (Deutsches Universalwörterbuch) (5. überarbeitete Aufgabe) (2003). Dudenverlag. Mannheim. Leipzig. Wien. Zürich.

5. Duden (2008): Wörterbuch der deutschen Idiomatik. 3., Mannheim: Dudenverlag.

6. Kempke, G. (1984):Handwörterbuch der deutschen Gegenwartssprache in zwei Bänden. Berlin: Akademie Verlag.

7. Paffen, K.A. (1980): Deutsch-russisches Satzlexikon. Leipzig: VEB Verlag Enzyklopädie.

\section{For the Symbolics and Semantics of "Fire" in German Phraseologisms}

M odebadze Tsisana

Iakob Gogebashvili State University, Telavi

\begin{abstract}
Phraseologisms represent the important and valuable legacy of the nation as they reflect not only cultural and historical ideology, but customs and traditions as w ell. The given research deals with the establishment of symbolics and semantic context of "Fire" in German phraseological units. German phraseological dictionaries are used as the research object.

Fire is a guarantee of improving human lives. It is the symbol of creative energy and destructive force as well. When the fire flame is under the human control it brings warmth, light and kindness. As soon as human reveals less care towards it the fire instantly turns into a dangerous, destructive force bringing death and collapse. Fire has different symbols in different religions. In Hinduism, fire is the symbol of creativity, absolution and demolition, in Islam birth and destruction, in Buddhism - wisdom, in Christianity it is the symbol of reanimation. Human ensures his/her welfare and safety with the help of fire. Fire is the symbol of native home, native land and family: Das heimische Feuer - native fire/hearth. Fire can figuratively express different emotions. Phraseologisms expressing love are mostly found out: Feuer der Liebe - fire of love. In a figurative way, fire also indicates such human traits as intrepidity, bravery, sympathy, goodwill, hatred, anger, enthusiasm and hope. Figuratively fire al so has the mission of increasing the power of trait.

According to the research, it becomes quite clear that the fire has both positive and negative connotations in phraseologisms, though the ones with positive connotation are more in number than the negative.
\end{abstract}

Key words: phraseologisms, symbolics, semantics, positive, negative.

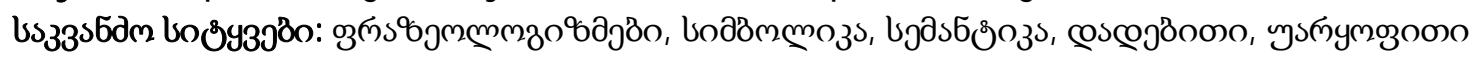

